\title{
Efeito antioxidante da diosmina: revisão integrativa
}

\section{The diosmin antioxidant effect: an integrative review}

Amanda Francielle Santos ${ }^{1}$, Rafaela Ribeiro Machado ${ }^{1}$, José Marden Mendes Neto², Míriam Geisa Virgens Menezes³, Gilmara Beatriz Andrade da Silva ${ }^{4}$, Sandra Lauton Santos ${ }^{5}$

${ }^{1}$ Curso de Enfermagem, Faculdade Estácio de Sergipe (FASE) - Aracaju (SE), Brasil.

${ }^{2}$ Ciências Fisiológicas, UFS - Aracaju (SE), Brasil.

${ }^{3}$ Curso de Enfermagem, UFS - Aracaju (SE), Brasil.

${ }^{4}$ Ciências da Saúde, Universidade Federal de Sergipe (UFS) - Aracaju (SE), Brasil.

${ }^{5}$ Bioquímica e Imunologia, UFS - Aracaju (SE), Brasil.

DOI: http://dx.doi.org/10.7322/abcshs.v43i3.1024

\section{RESUMO}

O estresse oxidativo está relacionado ao desenvolvimento de muitas enfermidades e atualmente tem-se utilizado a Diosmina visando reduzi-lo. O objetivo deste estudo é identificar através da literatura o efeito antioxidante da Diosmina. Trata-se de um estudo descritivo bibliográfico de revisão integrativa, realizada a partir artigos que abordassem o tema pesquisado publicados entre 2013 a 2017 oriundos das bases de dados eletrônicas PubMed e Bireme. Foram analisados 9 artigos. Os estudos avaliados evidenciam que com a utilização da Diosmina há uma elevação da quantidade no organismo de enzimas que combatem o estresse oxidativo como, catalase e outras. E também que ocorreu a redução do Malondialdeído. Concluise com esse estudo que a Diosmina contribui para o combate das lesões desencadeadas pelo desequilíbio no status redox que estimula o aumento de antioxidantes enzimáticos e inibe a formação de malondialdeído, assim colaborando para a diminuição de danos aos tecidos.

Palavras-chave: Antioxidantes; Diosmina; Estresse oxidativo.

\section{ABSTRACT}

Oxidative stress is related to the development of multiple diseases and some studies objective to identify the effect of natural substances in lesions caused by it. One of these substances is Diosmin, a flavonoid found in citric fruits. The objective of this study was to identify through the literature the anti-oxidative effect of Diosmin. It is a descriptive and bibliographic study of integrative revision, based on articles which discussed the researched topic published between 2013 and 2017 using the electronic database PubMed and Bireme. 9 articles were analyzed. The studies evaluated show that the use of Diosmin rises the activity of enzymes which fight against oxidative stress, as catalase, superoxide dismutase and glutathione. There was reduction of the concentration of malondialdehyde. It was proved with this study that Diosmin contributes to the fight against lesions caused by the unbalance between the redox status, stimulating the rise of activity of enzymatic anti-oxidants and reducing the production of malondialdehyde, collaborating to the reduction of damage to tissues generated by stress oxidative.

Keywords: Antioxidants; Diosmin; Oxidative stress. 


\section{INTRODUÇÃO}

As espécies reativas de oxigênio (EROs) constituem átomos, íns ou moléculas que contêm oxigênio com um elétron não-pareado em sua órbita externa, tais EROs possuem grande atividade reativa por serem moléculas instáveis participando das reações de oxidação-redução com outras moléculas encontradas nas células, tais como: lipídios, proteínas e DNA. As principais espécies reativas de oxigênio são: o ânion superóxido $\left(\mathrm{O}_{2}{ }^{-}\right)$, radical hidroxila ( $\left.\mathrm{HO}^{*}\right)$, peróxido de hidrogênio $\left(\mathrm{H}_{2} \mathrm{O}_{2}\right)$ e o peroxinitrito (ONOO-) os quais possuem atividade contrabalanceada e equilibrada pela atividade de agentes antioxidantes endógenos, tais como: as enzimas antioxidantes superóxido dismutase (SOD), catalase (CAT), glutationa peroxidade $(\mathrm{GPx})$ e a glutationa reduzida $(\mathrm{GSH})^{1,2}$.

As EROs são produzidas durante o processo metabólico no organismo humano, assim para gerar energia as reações de oxidação-redução são imprescindíveis. Essas reações caracterizam-se na transferência de elétrons de um reagente para outro, desse modo na molécula que doa elétrons há oxidação e a que recebe tem-se redução. No oxigênio (O2-) proveniente da respiração ocorre à redução, nesse processo de ganho de elétrons o O2 gera as espécies reativas pela redução de um elétron produzindo o ânion superóxido e pela de dois elétrons será formado o peróxido de hidrogênio, além disso, o ânion superóxido é formado nas mitocôndrias durante o processo de respiração celular³ .

O radical hidroxila é sintetizado a partir das reações químicas de Fenton e Haber-Weiss ${ }^{4}$, na primeira ocorre catalise de metais pesados, principalmente ferro e cobre que doam um terceiro elétron ao $\mathrm{H}_{2} \mathrm{O}_{2}$ e produzem o $\mathrm{HO}^{-}$, conforme a reação: $\mathrm{H}_{2} \mathrm{O}_{2}+\mathrm{Cu}^{+} /$ $\mathrm{Fe}^{+2} \rightarrow \mathrm{HO}^{-}+\mathrm{OH}^{-}+\mathrm{Cu}^{+2} / \mathrm{Fe}^{+3}$. Na reação de Haber-Weiss, o oxigênio molecular $\mathrm{O}_{2}$ reage com o $\mathrm{H}_{2} \mathrm{O}_{2}$ para formar como um dos produtos o $\mathrm{OH}^{-}$e o ânion superóxido $\mathrm{O}_{2}^{-}$, como mostra a seguinte reação: $\mathrm{O}_{2}^{\cdot-}+\mathrm{H}_{2} \mathrm{O}_{2} \rightarrow \mathrm{O}_{2}+{ }^{\cdot} \mathrm{OH}+\mathrm{OH}^{-}$.

O estresse oxidativo é a situação, na qual, ocorre desequilíbrio entre a produção de EROs e dos agentes antioxidantes endógenos para equilibrar o sistema, desencadeando múltiplas alterações celulares, como por exemplo, a lipoperoxidação da membrana, evento que prejudica o transporte através da bicamada lipídica, bem como alterações estruturais no DNA e ainda a oxidação de proteínas desencadeando prejuízo no funcionamento das mesmas. Esses eventos promovem o surgimento de algumas patologias, a despeito de algumas disfunções cardiovasculares e neurodegenerativas, além do surgimento de alguns tipos de câncer ${ }^{5,6}$.

Além do sistema antioxidante endógeno algumas moléculas possuem atividade biológica no estresse oxidativo, por influenciar as EROs. São as substâncias antioxidantes que podem inativá-las diretamente por meio de uma ligação molecular ou estimular a atividade das enzimas antioxidantes endógenas, as quais irão reequilibrar o status redox por inativar as EROs, um exemplo destas substâncias são: as vitaminas, o licopeno, os flavonóides, entre outros ${ }^{7-9}$.

Os flavonóides são polifenóis, encontrados em uma grande variedade de alimentos, como na soja, na uva, na cebola e nas frutas cítricas. A depender da estrutura química, tais moléculas podem ser classificadas como: antocianinas, flavonas, flavonois, auronas, cauconas, isoflavonas, flavononas, catequinas e dihidroflavonois. Possuem a capacidade de inativar as espécies reativas de oxigênio por desencadear aumento da atividade de algumas enzimas antioxidantes ou pela ligação direta com a EROs de modo a estabilizá-las, contribuindo para reequilibrar o status redox ${ }^{10,11}$.

A Diosmin (DIO), (diosmetin 7-O-rutinoside), uma flavona cítrica natural, com múltiplos efeitos: antioxidantes, anti-inflamatórios e anti-apoptóticos ${ }^{12}$. É uma medicação utilizada para a terapêutica de enfermidades como insuficiência venosa crônica e da doença hemorroidária ${ }^{13}$. A utilização DIO é segura em curto prazo, por até 3 meses, há descrição de alguns efeitos colaterais, como diarreia, cefaleia, dor epigástrica e abdominal, também está contraindicada no período da gravidez e lactação ${ }^{14}$.

$\mathrm{O}$ efeito antioxidante da DIO pode ser atribuído à remoção de ânions superóxido, através da ligação direta, ao aumento da biodisponibilidade do óxido nítico, além de estimular a redução da peroxidação lipídica no plasma e nos tecidos renal e cardiovascular. Com isso a substância poderá contribuir na redução das lesões desencadeadas nas membranas das células, no DNA e nas proteínas pelas EROs ${ }^{15}$.

Visto que, o estresse oxidativo causa patologias de grande impacto e comuns na sociedade. O estudo justifica-se pela necessidade em melhor conhecer a ação antioxidante da Diosmina na reestruturação do status redox celular, visando confirmar a eficácia desse flavonóide, a fim de buscar alternativas para o controle dos danos oxidativos. O objetivo deste estudo foi identificar através da literatura o efeito antioxidante da Diosmina.

\section{MÉTODO}

Foi realizada uma revisão integrativa descritiva qualitativa em artigos internacionais oriundos das bases de dados eletrônicas PubMed e Bireme durante os meses de março a julho de 2017. Foram selecionados para análises artigos de 2013 a 2017. A detecção ocorreu mediante a utilização dos seguintes descritores: "Antioxidante", "Diosmina" e "Estresse Oxidativo". Combinados com o operador booleano "and". Critérios de inclusão do estudo: trabalhos que se enquadraram na categoria artigo científico; em português e língua estrangeira (inglês ou espanhol); publicados entre 2013 a 2017, que abordassem os termos citados anteriormente. Foram excluídos do estudo: monografias; artigos de revisão; anais de eventos; dissertações; teses; livros; além de artigos fora do período estabelecido no estudo. Após a seleção foram analisados 09 artigos e posteriormente submetidos à produção de fichamentos dos conteúdos relevantes à temática. A caracterização dos estudos e os resultados acerca da Diosmina foram relatados e expostos na Figura 1.

\section{RESULTADOS E DISCUSSÃO}

A maioria dos estudos publicados que avaliaram o potencial da Diosmina no combate ao estresse oxidativo, nos últimos cinco 
anos, foram realizados na Índia no ano de 2013. Os animais para os experimentos foram ratos Wistar machos com patologias cardíaca, hepática ou renal. Os resultados indicam que a Diosmina, um flavonóide, apresenta atividade antioxidante por variados mecanismos de ação ${ }^{16-23}$, contribuindo para a redução das lesões cardíacas, hepáticas e renais induzidas em muitos modelos de experimentação animal (Tabela 1).

Múltiplos modelos de experimentação animal podem ser utilizados para se estudar os mecanismos fisiopatológicos existentes nas mais variadas doenças. Nos estudos analisados, todos avaliaram o potencial da Diosmina em reestabelecer o equilíbrio no status redox em situações de lesões desencadeadas pela sobreposição da produção de espécies reativas em função da defesa antioxidante endógena.

A implementação do tratamento com uma substância antioxidante exógena, poderá desencadear um efeito antioxidante explicado por alguns mecanismos, seja funcionando como um sequestrador de EROs, ou molécula que se liga as espécies reativas e as estabilizam, ou ainda o agente antioxidante exógeno poderá aumentar a atividade das enzimas antioxidantes ativando-as em sítios estruturais específicos.

Durante condições de estresse oxidativo a defesa antioxidante natural do corpo torna-se prejudicada, resultando no declínio da atividade das várias enzimas antioxidantes, tais como, superóxido dismutase intracelular, catalase e glutationareduzida, gerando várias anormalidades celulares ${ }^{24}$. Essas enzimas funcionam juntas para eliminar os radicais livres formados e qualquer compromentimento das mesmas poderá resultar em graves lesões ao DNA, lipídios e proteínas².

Os estudos evidenciaram que um dos mecanismos de ação antioxidante da Diosmina é a elevação na atividade das enzimas que degradam as EROs como, a catalase, a glutationa reduzida, a glutationa peroxidase, a superóxido dismutase e a glutationa transferase (Tabela 1).

$\mathrm{O}$ oxigênio produz muitas espécies reativas (EROs) radicais livres e espécies não radicais. Quando o oxigênio absorve energia, cria uma espécie eletronicamente excitada nomeada oxigênio singlete $\left(\mathrm{O}_{2}\right)$. Já na redução do oxigênio à água, ocorre o desenvolvimento do radical superóxido $\left(\mathrm{O}_{2}^{*}\right)$, peróxido de hidrogênio $\left(\mathrm{H}_{2} \mathrm{O}_{2}\right)$ e radical hidroxila $(\cdot \mathrm{OH})$, que podem interagir e provocar danos a diferentes estruturas celulares ${ }^{25-27}$.

Com a formação das EROs ocorre o acionamento do sistema antioxidante endógeno, ou seja, com a formação dos substratos as enzimas antioxiantes, SOD, CAT, GPx e GSH são ativadas convertendo tais espécies em moléculas estáveis. A CAT desencadeia a conversão do peróxido de hidrogênio em água e oxigênio, obedecendo a seguinte reação: $2 \mathrm{H}_{2} \mathrm{O}_{2} \rightarrow 2 \mathrm{H}_{2} \mathrm{O}+\mathrm{O}_{2}$, outra enzima,

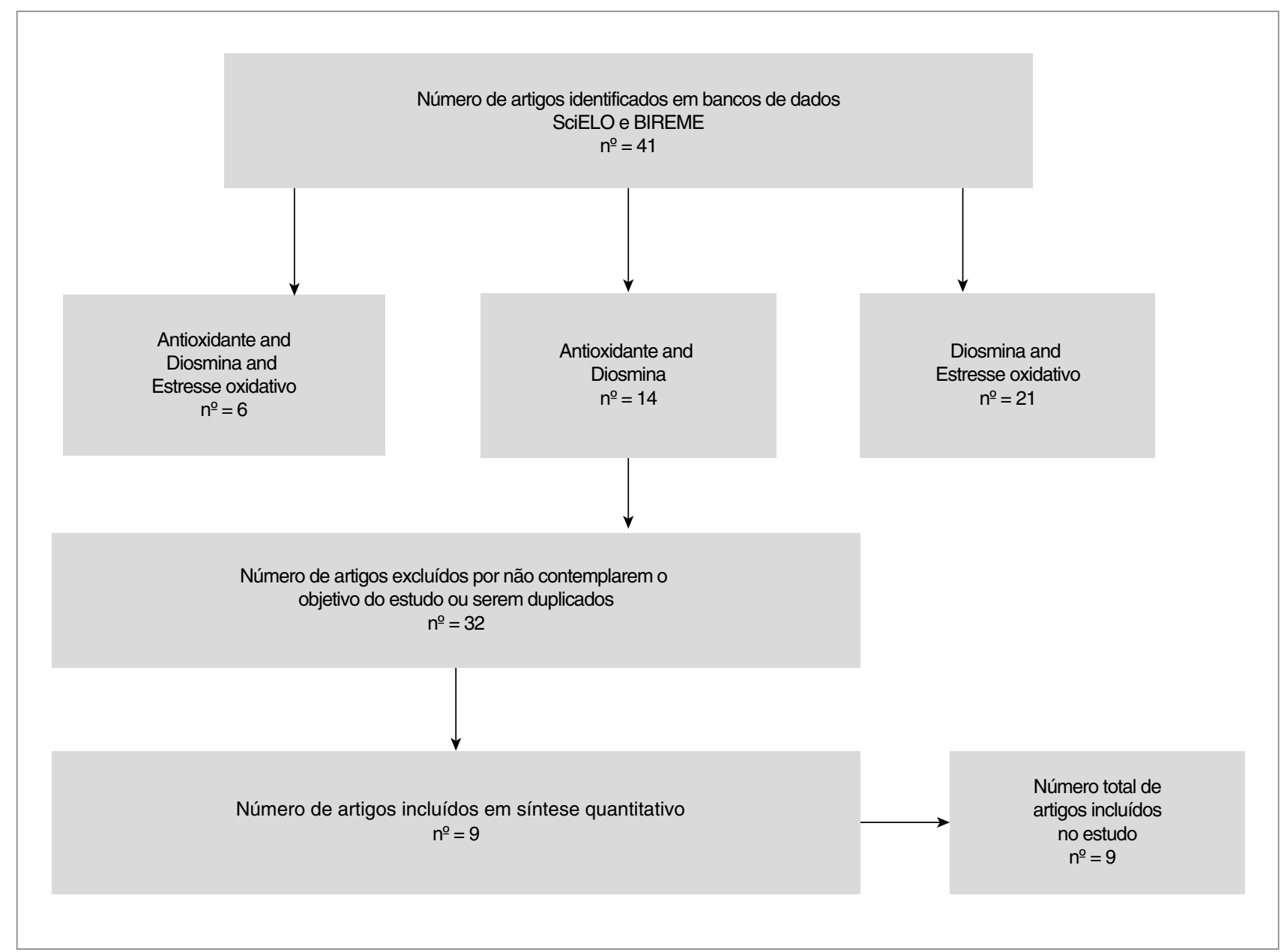

Figura1: Processo de pesquisa e seleção dos artigos utilizados no estudo. 
a SOD, dismuta o ânion superóxido em peróxido de hidrogênio e oxigênio, peróxido de hidrogênio formado pela atividade da SOD será degradado pela CAT. A enzima GPx desencadeia a formação de duas moléculas de água a partir do peróxido de hidrogênio, porém é uma enzima que necessita da glutationa reduzida (GSH) para exercer sua atividade ${ }^{28}$.
A transformação da LDL pode ser resultado da sua oxidação por espécies reativas de oxigênio na presença de metais de transição como cobre e ferro, que começa com a peroxidação dos ácidos graxos polinsaturados da $\mathrm{LDL}$, em qualquer membrana biológica $\left(\mathrm{RH}+\mathrm{OH}^{\bullet} \rightarrow\right.$ $\left.\mathrm{H}_{2} 0+\mathrm{R} \cdot\right)$. A peroxidação lipídica caso não contida pode gerar reação serial, uma vez que, os radicais desenvolvidos reagem novamente com

Tabela 1: Caracterização dos estudos experimentais que mostram a ação da Diosmina na redução do estresse oxidativo, 2013 a 2017.

\begin{tabular}{|c|c|c|c|c|}
\hline Autor/ ano & Local & $\begin{array}{c}\text { Patologia da } \\
\text { amostra }\end{array}$ & Amostra & Mecanismo de ação antioxidante da Diosmina \\
\hline $\begin{array}{l}\text { Tahir et al., } \\
2013^{12}\end{array}$ & Índia & $\begin{array}{l}\text { Câncer } \\
\text { Hepático }\end{array}$ & $\begin{array}{l}\text { Ratos } \\
\text { Wistar } \\
\text { fêmeas }\end{array}$ & $\begin{array}{l}\text { Os resultados do estudo de curto prazo mostraram que a Diosmina é muito } \\
\text { potente na redução do estresse oxidativo e eventos tumorais iniciais induzidos por } \\
\text { 2-acetylaminofluorene. O tratamento da Diosmina aumenta significativamente a } \\
\text { atividade de glutationatransferase }(0,057 \pm 0,006, p<0,05) \text {, glutationaperoxidase } \\
(191,9 \pm 14,89, p<0,05) \text {, glutationaredutas e }(240,6 \pm 26,22, p<0,05) \text { e catalase } \\
(17,09 \pm 1,5, p<0,01) .\end{array}$ \\
\hline $\begin{array}{l}\text { Ahmed et al., } \\
2016^{16}\end{array}$ & Índia & $\begin{array}{l}\text { Diabetes } \\
\text { tipo } 2\end{array}$ & $\begin{array}{l}\text { Ratos } \\
\text { Wistar }\end{array}$ & $\begin{array}{c}\text { O tratamento com Diosmina por um período de } 4 \text { semanas reduziu o estresse oxidativo } \\
\text { mediado pela hiperglicemia. Houve maior atividade do superóxido desmutase enzimático } \\
(85,24 \pm 2,72)(p<0,01) \text {, catalase }(34,91 \pm 1,37)(p<0,01) \text { e glutationaredutase não } \\
\text { enzimático }(83,94 \pm 1,80)(p<0,01) \text { em níveis antioxidantes quando comparados aos } \\
\text { grupos de controle e reduziua peroxidação lipídica por inibir a formação as substâncias } \\
\text { reativas ao ácido tiobarbitúrico induzidos por aloxano }(p<0,05) \text {. }\end{array}$ \\
\hline $\begin{array}{l}\text { Tanrikulu et al., } \\
2013^{17}\end{array}$ & Turquia & $\begin{array}{l}\text { Isquemia } \\
\text { hepática }\end{array}$ & $\begin{array}{l}\text { Ratos } \\
\text { Wistar } \\
\text { Albino } \\
\text { fêmeas }\end{array}$ & $\begin{array}{l}\text { Os níveis de xantina oxidase e malondialdeídoplasmático foram menores nos } \\
\text { grupos de tratamento com Diosmina do que no grupo de controle, porém os níveis } \\
\text { plasmáticos de glutationaperoxidase foram maiores ( } p<0,05 \text { para todos). Os níveis } \\
\text { de malondialdeído nos tecidos foram menores no grupotratamento do que grupo de } \\
\text { controle, mas no tecido glutationaperoxidase, superóxido dismutase, catalase e os } \\
\text { níveis xantina oxidase foram maiores. E os níveis de glutationaperoxidase, foram } \\
\text { significantemente altos em ambos os tratamentos grupos. }\end{array}$ \\
\hline $\begin{array}{l}\text { Queenthy; John, } \\
2013^{18}\end{array}$ & Índia & $\begin{array}{l}\text { Infarto do } \\
\text { miocárdio } \\
\text { Induzido. }\end{array}$ & $\begin{array}{l}\text { Ratos } \\
\text { Wistar } \\
\text { machos }\end{array}$ & $\begin{array}{l}\text { O pré-tratamento com Diosmina forneceu cardioproteção, evitando o acúmulo de } \\
\text { lipídios por meio de efeitos anti-hiperlipidêmicos de radicais livres. Os efeitos de } \\
\text { eliminação de radicais livres e anti-hiperlipidêmicos são os motivos dos efeitos } \\
\text { cardioprotetores da Diosmina. O estudo constatou a capacidade da Diosmina mesmo } \\
\text { em baixa dosagem na eliminação o superóxido e radicais livres de hidroxilo. }\end{array}$ \\
\hline $\begin{array}{l}\text { Rehman et al., } \\
2013^{19}\end{array}$ & Índia & $\begin{array}{l}\text { Toxicidade } \\
\text { renal } \\
\text { Por } \\
\text { Tricloroetileno }\end{array}$ & $\begin{array}{l}\text { Ratos } \\
\text { Wistar } \\
\text { machos }\end{array}$ & $\begin{array}{l}\text { O tratamento com Diosmina rediziu a toxidade renal induzida por Tricloroetileno } \\
\text { mantendo a integridade da membrana celular renal. O co-tratamento com Diosmina } \\
\text { reduziu o estresse oxidativo, restaurando os níveis de enzimas antioxidantes. } \\
\text { Diosmina significativamente recuperou a atividades de glutationatransferase }(p<0,01) \text {, } \\
\text { glutationaperoxidase }(p<0,01) \text { e as enzimas antioxidantes glutationaredutase } \\
\text { ( }<<0,01) \text {. O tratamento com Diosmina foi eficaz em aumentar a atividade de } \\
\text { superóxido dismutase }(p<0,05) \text { e catalase }(p<0,05) \text { ao normal nas duas doses. }\end{array}$ \\
\hline $\begin{array}{l}\text { Senthamizhselvan } \\
\text { et al., } 2014^{20}\end{array}$ & Índia & $\begin{array}{l}\text { Isquemia do } \\
\text { miocárdio }\end{array}$ & $\begin{array}{l}\text { Ratos } \\
\text { Wistar } \\
\text { machos }\end{array}$ & $\begin{array}{l}\text { O pré-tratamento de Diosmina evitou os prejuízos causados a função cardíaca, } \\
\text { o estresse oxidativo e a apoptose associada à reperfusão no coração de controle pelo } \\
\text { seu papel antioxidante. Assim, as espécies reativas de oxigênio, como superóxido } \\
\text { e hidroxilo foram efetivamente diminuídos pela substância. A Diosmina } \\
(50 \text { e } 100 \mathrm{mg} / \mathrm{kg}) \text { significativamente }(p<0,05) \text { eleva as atividades dessas enzimas e o } \\
\text { nível de glutiatonaredutase, superoxidodismutase, catalase e glutationaperoxidase. }\end{array}$ \\
\hline $\begin{array}{l}\text { Arab et al., } \\
2015^{21}\end{array}$ & $\begin{array}{l}\text { Estados } \\
\text { Unidos }\end{array}$ & $\begin{array}{l}\text { Lesões da } \\
\text { mucosa } \\
\text { gástrica }\end{array}$ & $\begin{array}{l}\text { Ratos } \\
\text { Wistar } \\
\text { machos }\end{array}$ & $\begin{array}{l}\text { O pré-tratamento com Diosmina durante uma semana protegeu significativamente } \\
\text { do estresse oxidativo como evidenciado pela redução do malondialdeído e aumento } \\
\text { da atividade da glutationaredutase, glutationaperoxidase e capacidade total } \\
\text { antioxidante. Atenuou ainda o dano grosseiro da mucosa gástrica e diminuiu a área } \\
\text { de lesões gástricas. Essas ações favoráveis foram mediadas através da supressão } \\
\text { da inflamação gástrica e do estresse oxidativo, principalmente através da inibição } \\
\text { de o fator nuclear kappa, houve aumento das defesas da mucosa pela ação de } \\
\text { antioxidantes e citoprotetoras. }\end{array}$ \\
\hline $\begin{array}{l}\text { Schlottfeldt et al., } \\
2015^{22}\end{array}$ & Brasil & $\begin{array}{c}\text { Lesão } \\
\text { renal por } \\
\text { Anfotericina B }\end{array}$ & $\begin{array}{l}\text { Ratos } \\
\text { Wistar } \\
\text { machos }\end{array}$ & $\begin{array}{c}\text { A aplicação de Anf-B levou a diminuição da função renal e lesão tubular com } \\
\text { envolvimento de espécies de oxigênio reativo no mecanismo de lesão. } \\
\text { O pré-condicionamento com a Diosmina e hesperidina mostrou proteção antioxidante. } \\
\text { O grupo de animais em uso da anfotericina } B+\text { diosmina obteve redução } \\
\text { significativa dos peróxidos urinários }(p<0,05) \text {. }\end{array}$ \\
\hline $\begin{array}{l}\text { Hasan et al., } \\
2017^{23}\end{array}$ & Egito & $\begin{array}{l}\text { Fibrose } \\
\text { hepatica } \\
\text { induzida } \\
\text { porradiação }\end{array}$ & $\begin{array}{l}\text { Ratos } \\
\text { Wistar } \\
\text { machos }\end{array}$ & $\begin{array}{c}\text { O tratamento com Diosmina atenuou o estresse oxidativo, aprimorou as defesas } \\
\text { antioxidantes, diminuiu as respostas inflamatórias hepáticas, evidenciando dessa } \\
\text { forma o potencial hepatoprotetor e anti-fibrótico da mesma contra fibrose hepática } \\
\text { induzida por radiação. O tratamento de ratos irradiados com Diosmina conseguiu } \\
\text { uma redução de } 1,9 \text { vezes no malondialdeído quando comparado ao grupo } \\
\text { sem a Diosmina. Além disso,o tratamento com Diosmina conseguiu restaurar } \\
\text { a defesa antioxidante como evidenciado pelo aumento }(p<0,05) \text { nas atividades } \\
\text { glutationaperoxidase e catalase e glutationaredutase. }\end{array}$ \\
\hline
\end{tabular}


o oxigênio compondo um peroxirradical de ácido graxo $\left(\mathrm{R}^{\bullet}+\mathrm{O} 2 \rightarrow\right.$ ROO*) que reage com um ácido graxo, produzindo uma reação em cadeia e formando malonaldeido ( $\left.\mathrm{ROO} *+\mathrm{RH} \rightarrow \mathrm{ROOH}+\mathrm{R}^{*}\right)^{28}$.

Avaliando os feitos da DIO no miocárdio de ratos infartados observou-se a diminuição das alterações eletrocardiográficas e o grau de peroxidação lipídica ${ }^{3}$. Três dos nove estudos avaliados afirmaram que ocorreu a redução do malondialdeído. A concentração de malondialdeído é considerada um índice para a mensuração do estresse oxidativo, por ser o produto final da peroxidação dos lipídeos, ou seja, da peroxidação da membrana nos mais variados tecidos, que é aumentado em situações de maior estresse oxidativo ${ }^{29}$. Sua redução com a utilização da Diosmina mostra que ela contribui para a redução dos danos celulares originados da ação da ROS.

\section{CONCLUSÃO}

A Diosmina tem grande potencial para reduzir as lesões desencadeadas pelo desequilíbrio entre a produção de espécies reativas e a atuação dos sistemas antioxidantes. A substância possui como principais mecanismos de ação: a estimulação do aumento de antioxidantes enzimáticos e a redução da formação de malondialdeído, assim colaborando para a diminuição de danos ao tecido cardíaco, hepático e renal causados por espécies reativas.

Pode ser considerado um fator limitante do estudo ter apenas um trabalho nacional e a pouca quantidade de pesquisas avaliadas. É recomendada a realização de estudos nacionais e internacionais para a avaliação da Diosmina no combate ao estresse oxidativo.

\section{REFERÊNCIAS}

1. Jacob KD, NorenHooten N, Trzeciak AR, Evans MK. Markers of oxidant stress that are clinically relevant in aging and age-related disease. Mech Ageing Dev. 2013;134(3-4):139-57. http://dx.doi.org/10.1016/j.mad.2013.02.008

2. Gasparovic AC, Jaganjac M, Mihaljevic B, Sunjic SB, Zarkovic N Assays for the measurement of lipid peroxidation. Methods Mol Biol. 2013;965:283-96 http://dx.doi.org/10.1007/978-1-62703-239-119

3. McCord JM. The evolution of free radical and oxidative stress. Am J Med. 2000;108(8):652-9. https://dx.doi.org/10.1016/S0002-9343(00)00412-5

4. Sterba M, Popelová O, Vávrová $A$, Jirkovský E, Kovaříková $P$, Gerš $\mathrm{V}$, et al. Oxidative stress, redox signaling, and metal chelation in anthracyclinecardiotoxicity and pharmacological cardioprotection. Antioxid Redox Signal. 2013;18(8):899-929. https://dx.doi.org/10.1089/ars.2012.4795

5. Nordberg J, Arner ESJ. Reactive oxygen species, antioxidant and the mammalian thioredoxin system. Free Rad Biol and Med. 2001;31(11):1287-312

https://dx.doi.org/10.1016/S0891-5849(01)00724-9

6. Barbosa KBF, Costa NMB, Alfenas RCG, Paula SO, Minim VPR, Bressan J. Estresse oxidativo: conceito, implicações e fatores modulatórios. Rev Nutr. 2010;23(4):629-43 http://dx.doi.org/10.1590/S1415-52732010000400013

7. McCord, JM.The evolution of free radicals and oxidative stress. Am J Med. 2000;108(8):652-9. http://dx.doi.org/10.1016/S0002-9343(00)00412-5

8. Betteridge, DJ. What is oxidative stress? Metabolism. 2000;49(2 Suppl 1):3-8

http://dx.doi.org/10.1016/S0026-0495(00)80077-3

9. Halliwell B. Oxidants and human disease: some new concepts. FASEB J. 1987;1(5):358-64.

https://dx.doi.org/10.1096/fasebj.1.5.2824268

10. Barreiros ALBS, David JM, David JP. Estresse oxidativo: relação entre geração de espécies reativas e defesa do organismo. Quím Nova. 2006:29(1):113-23. http://dx.doi.org/10.1590/S0100-40422006000100021

11. Cook NC, Sammans S. Flavonoids- Chemistry, metabolism, cardioprotective effects, and dietary sources. J Nutr Biochem. 1996;7:66-76.

https://dx.doi.org/10.1016/S0955-2863(95)00168-9
12. Tahir M, Rehman MU, Lateef $A$, Khan $A Q$, Khan R, Qamar W, et al. Diosmin abrogate schemically induced hepatocarcinogenesis via alleviation of oxidative stress, hyperproliferative and inflammatory markers in murine model. Toxicol Lett. 2013;220(3):205-18. http://dx.doi.org/10.1016/j.toxlet.2013.04.004

13. Tamamoto LC1, Schmidt SJ, Lee SY. Sensory profile of a model energy drink with varying levels of functional ingredients-caffeine, ginseng, and taurine. J Food Sci. 2010;75(6):S271-8. https://dx.doi.org/10.1111/j.1750-3841.2010.01655.x

14. Craig WJ. Health promoting properties of common herbs. Am J Clin Nutr. 1999;70(Suppl.3):491s-9 https://dx.doi.org/10.1111/10.1093/ajcn/70.3.491s

15. Silambarasan T, Raja B. Diosmin, a bioflavonoid reverses alterations in blood pressure, nitric oxide, lipid peroxides and antioxidant status in DOCA-salt induced hypertensive rats. Eur $J$ Pharmacol. 2012;679(1-3):81-9. dx.https://dx.doi.org/10.1016/j.ejphar.2011.12.040

16. Ahmed S, Mundhe N, Borgohain M, Chowdhury L, Kwatra $\mathrm{M}$, Bolshette $\mathrm{N}$, et al. Diosmin modulates the NF-kB signal transduction pathways and downregulation of various oxidative stress markers in alloxan induced diabetic nephropathy. Inflammation. 2016;39(5):1783-97. http://dx.doi.org/10.1007/s10753-016-0413-4

17. Tanrikulu Y, Sahin M, Kismet K, Kilicoglu SS, Devrim E, Tanrikulu CS, et al. The protective effect of diosmin on hepatic ischemia reperfusion injury: an experimental study. Bosn J Basic Med Sci. 2013;13(4):218-24.

http://dx.doi.org/10.17305/bjbms.2013.2305

18. Queenthy SS, John B. Diosmin exhibits anti-hyperlipidemic effects in isoproterenol induced myocardial infarcted rats. Eur $J$ Pharmacol. 2013;718(1-3):213-8.

http://dx.doi.org/10.1016/j.ejphar.2013.08.031

19. Rehman MU, Tahir M, Quaiyoom Khan A, Khan R, Lateef A, Hamiza OO, et al. Diosmin protects against trichloroethyleneinduced renal injury in Wistar rats: plausible role of p53, Bax and caspases. Br J Nutr. 2013;110(4):699-710. dx.https://dx.doi.org/10.1017/S0007114512005752

20. Senthamizhselvan, Manivannan J, Silambarasan T, Raja B. Diosmin pretreatment improves cardiac function and suppresses oxidative stress in rat heart after ischemia/reperfusion. Eur $J$ Pharmacol. 2014;736:131-7. http://dx.doi.org/10.1016/j.ejphar.2014.04.026 
21. Arab HH, Salama SA, Omar HA, Arafael-SA, Maghrabi IA. Diosmin protects against ethanol-induced gastric injury in rats: novel antiulcer actions. PloS One. 2015;10(3):e0122417. https://dx.doi.org/10.1371/journal.pone.0122417

22. Schlottfeldt FS, Fernandes SM, Martins DM, Cordeiro P, Fonseca $\mathrm{CD}$, Watanabe $\mathrm{M}$, et al. Prevenção da nefrotoxicidade da anfotericina B por meio do uso de fitomedicamentos. Rev Esc Enferm USP. 2015;49(esp)74-9. http://dx.doi.org/10.1590/S0080-623420150000700011

23. Hasan HF, Abdel-Rafei MK, Galal SM. Diosmin attenuates radiation-induced hepatic fibrosis by boosting PPAR- $\gamma$ expression and hampering miR-17-5p-activated canonical Wnt- $\beta$-catenin signaling. Biochem Cell Biol. 2017;95(3):400-14. https://dx.doi.org/10.1139/bcb-2016-0142

24. Bondeva T, Wolf G. Reactive oxygen species in diabetic nephropathy: friend or foe? Nephrol Dial Transplant. 2014;29(11):1998-2003

https://dx.doi.org/10.1093/ndt/gfu037
25. Hasanoğlu E1, Altan N, Sindel S, Ongun CO, Bali M, Altintaş E. The relation ship between erythrocyte superoxide dismutase activity and plasma levelsof some trace elements (AL, CU, ZN) of dialysis patients. Gen Pharmacol. 1994;25(1):107-10. https://dx.doi.org/10.1016/0306-3623(94)90018-3

26. Richard MJ,ArnaudJ,Jurkovitz C, Hachache T, Meftahi H, Laporte $F$, et al. Trace elements and lipid peroxidation abnormalities in patients with chronic renal failure. Nephron 1991;57:10-15. https://dx.doi.org/10.1159/000186208

27. Abdalla DSP. Antioxidantes. Conceitos básicos e perspectivas terapêuticas. ARS Cvrandi. 1993;26:141-64

28. Halliwell B, Gutteridge JM, Cross CE. Free radicals, antioxidants, and human disease: where are we now? J Lab Clin Med. 1992;119(6):598-620.

29. Kim MJ, Lim Y. Protective effect of short-term genistein supplementation on the early stage in diabetes-induced renal damage. Mediators Inflamm. 2013;2013:510212. https://dx.doi.org/10.1155/2013/510212 